\title{
A three-dimensional printed customized bolus: adapting to the shape of the outer ear
}

\author{
Gorka Gomez ${ }^{1}$, Montserrat Baeza ${ }^{2}$ Juan Carlos Mateos², Jose Antonio Rivas², Florencio Javier Luis Simon ${ }^{2}$, \\ Diego Mesta Ortega ${ }^{3}$, María de los Ángeles Flores Carrión ${ }^{4}$, Eleonor Rivin del Campo ${ }^{5}$, Tomas Gómez-Cía ${ }^{6,7}$, \\ Jose Luis Lopez Guerra, \\ ${ }^{1}$ Biomedical Informatics, Biomedical Engineering and Health Economy, Institute of Biomedicine of Seville (IBIS)/ \\ /Virgen del Rocío University Hospital/CSIC/University of Seville, Seville, Spain \\ ${ }^{2}$ Radiation Physics, University Hospital Virgen del Rocio, Seville, Spain \\ ${ }^{3}$ Department of Radiation Oncology, University Hospital Virgen del Rocio, Seville, Spain \\ ${ }^{4}$ Department of Radiation Oncology, Juan Ramón Jiménez Hospital, Huelva, Spain \\ ${ }^{5}$ Department of Radiation Oncology, Tenon University Hospital, Hôpitaux Universitaires Est Parisien, \\ Sorbonne University Medical Faculty, Paris, France \\ ${ }^{6}$ Instituto de Biomedicina de Sevilla (IBIS/HUVR/CSIC/Universidad de Sevilla), Seville, Spain \\ ${ }^{7}$ Department of Plastic Surgery, University Hospital Virgen del Rocio, Seville, Spain
}

\begin{abstract}
Background: The skin-sparing effect of megavoltage-photon beams in radiotherapy (RT) reduces the target coverage of superficial tumours. Consequently, a bolus is widely used to enhance the target coverage for superficial targets. This study evaluates a three-dimensional (3D)-printed customized bolus for a very irregular surface, the outer ear.

Materials and methods: We fabricated a bolus using a computed tomography (CT) scanner and evaluated its efficacy. The head of an Alderson Rando phantom was scanned with a CT scanner. Two 3D boluses of 5- and 10-mm thickness were designed to fit on the surface of the ear. They were printed by the Stratasys Objet260 Connex 3 using the malleable "rubber-like" photopolymer Agilus. CT simulations of the Rando phantom with and without the 3D and commercial high density boluses were performed to evaluate the dosimetric properties of the 3D bolus. The prescription dose to the outer ear was 50 Gy at 2 Gy/fraction.

Results: We observed that the target coverage was slightly better with the 3D bolus of $10 \mathrm{~mm}$ compared with the commercial one $\left(D_{98 \%} 98.2 \%\right.$ vs. $\left.97.6 \%\right)$. The maximum dose was reduced by $6.6 \%$ with the $3 \mathrm{D}$ bolus and the minimum dose increased by $5.2 \%$ when comparing with the commercial bolus. In addition, the homogeneity index was better for the 3D bolus ( 0.041 vs. 0.073).

Conclusion: We successfully fabricated a customized 3D bolus for a very irregular surface. The target coverage and dosimetric parameters were at least comparable with a commercial bolus. Thus, the use of malleable materials can be considered for the fabrication of customized boluses in cases with complex anatomy.

Key words: three-dimensional printing; bolus; radiation therapy; auricular skin

Rep Pract Oncol Radiother 2021;26(2):211-217
\end{abstract}

Address for correspondence: Jose Luis Lopez Guerra, M.D., Ph.D. Department of Radiation Oncology, Virgen del Rocío University Hospital. Manuel Siurot avenue, s/n. 41013, Seville (Spain), tel: (+34) 95501 2105, fax: (+34) 95501 2111; e-mail: chanodetriana@yahoo.es

This article is available in open access under Creative Common Attribution-Non-Commercial-No Derivatives 4.0 International (CC BY-NC-ND 4.0) license, allowing to download articles and share them with others as long as they credit the authors and the publisher, but without permission to change them in any way or use them commercially 


\section{Introduction}

Radiation therapy (RT) is one of the most common treatments for cancer. RT effectively treats cancer by using high-energy beams to pinpoint and destroy cancer cells. The interaction between incident high energy photons and electrons from the body tissue produce secondary electrons. Chemical reactions induced by these secondary electrons are known as major molecular mechanism of RT. Due to kinetic energy transmitted from the photon, deposition of the secondary electron occurs after a few millimeters of travel in the forward direction from the interaction point. Because there are no interaction points upstream from the surface to produce secondary electrons, RT dose of the surface dose is low [1]. Electron conformal therapy has been used for treating superficial cancers, owing to the fact that it results in a specific dose distribution. In addition, the electron beam have a sharp distal fall off, reducing unnecessary irradiation to the underlying healthy normal tissue. However, inhomogeneous dose delivery can occur in the target volume owing to irregularities in the skin surface and varying target depths [2]. Consequently, a bolus is widely used to enhance the target coverage for superficial targets.

Several studies have demonstrated the clinical efficacy of the bolus in different settings $[3,4]$. The University of Texas M.D. Anderson Cancer Center [3] reported the outcome of 70 patients with non-metastatic angiosarcoma. Fifty out of 70 patients underwent RT. Tissue equivalent bolus material was applied to the skin surface to ensure adequate dose to the entire thickness of the dermal tissues and to minimize the dose to underlying brain tissue. They conclude that custom wax bolus or another similar device may be necessary to provide adequate dose build up to the skin surface. An Australian study [4] explored the effect of the chest wall bolus technique on chest wall recurrence. This was a retrospective cohort study of 254 patients treated with adjuvant postmastectomy RT between 1993 and 2003. In all, 143 (56\%) patients received RT with whole chest wall bolus, 88 (35\%) patients with a parascar bolus, and 23 (9\%) with no bolus. On multivariate analysis, whole chest wall bolus was found to be a significant predictor for early cessation of RT resulting from acute skin toxicity.
In addition, a failure to complete RT because of acute skin toxicity was associated with chest wall recurrence.

A commercial bolus cannot easily be applied on irregular surfaces, where unwanted air gaps exist between the bolus and body surface, thereby resulting in decreased surface doses. In order to solve this issue, three-dimensional boluses (3D boluses) have been successfully fabricated and applied to Rando phantoms with the use of 3D printing technologies [5]. This technique has been used for a variety of medical applications, including educational training tools [6]. In this regard, the application of 3D printing technology to radiation oncology has formed a strong research focus [7, 8]. Burleson et al. [7] designed a custom 3D bolus to treat the Rando phantom nose. The agreement between measured and calculated values was good, with $86.5 \%$ of data points passing gamma requirements of $2 \mathrm{~mm}$ distance to agreement and $5 \%$ dose difference. In this context, we explore the $\mathrm{RT}$ application possibility of a $3 \mathrm{D}$ printed bolus made of malleable material for the very irregular shape of the outer ear.

\section{Materials and methods}

\section{Scanning and printing procedure}

The head of an Alderson Rando phantom (The Phantom Laboratory, Salem, NY, USA) was utilized for the fabrication process. Next, the image data of the phantom were acquired with the use of Toshiba Aquileon LB (Toshiba Medical Systems Corporation) and exported to the workstation. The image data were reconstructed, and a customized 3D bolus was designed and fabricated by a Stratasys Objet260 Connex3 using PolyJet technology (Stratasys Ltd., Eden Prairie, MN, USA) with the malleable 'rubber-like' photopolymer Agilus (Stratasys, Eden Prairie, MN, USA). 3D boluses of 2 to $10 \mathrm{~mm}$ thickness were designed to fit onto the ear. The case was immobilized using a standard S-frame with a thermoplastic mask and clear headrests. Computed tomography (CT) simulations of the Rando phantom with and without the 3D and commercial high density boluses were performed to evaluate the dosimetric properties of the $3 \mathrm{D}$ boluses. The CT images were acquired with a slice thickness of 2.5 $\mathrm{mm}$ contiguously and the outer ear was delineated as the target $(15 \mathrm{cc})$. 


\section{Treatment planning}

Intensity-modulated RT plans were generated in the Eclipse treatment planning system version 10.0 (Varian Medical System, Palo Alto, CA, USA) with the use of the Dose Volume Optimizer (DVO) optimization algorithm v.10.0 and the calculation Anisotropic Analytical algorithm (AAA) v.10.0. The prescription dose to the target (outer ear) was $50 \mathrm{~Gy}$ at $2 \mathrm{~Gy} /$ fraction. The treatment machine was a Clinac DHX, 120 MLC millennium with $6 \mathrm{MV}$ beam energy. Dosimetric parameters of 6 plans [ 2 without bolus, 1 with commercial bolus, and 3 (5-mm, 10-mm, and 10-mm thickness + gel) with 3D printed bolus] were assessed (Tab. 1). The RT plans consisted of 5 sliding window Intensity-Modulated Photon-Beam Therapy (IMRT) fields at gantry $0^{\circ}, 35^{\circ}, 70^{\circ}, 105^{\circ}$ and $140^{\circ}$. The collimator was set to $358^{\circ}$ and the dose rate was $300 \mathrm{um} / \mathrm{min}$. The objective functions used were adapted to each case to get the optimal results according to the clinical practice.

To ensure adequate dose coverage, two approaches were "applied", depending on whether or not there was a bolus. The plans with a bolus were prescribed to the mean dose (Dmean) according ICRU 83 [9]. A virtual target volume without a bolus was created excluding the $3 \mathrm{~mm}$ closest to the skin surface. For the plan without a bolus, the goal was to cover $95 \%$ of the virtual target covered by $95 \%$ of the prescription dose. The following dosimetric parameters were estimated for all cases: maximal dose (Dmax), Dmean, minimum dose (Dmin), V95\% (volume receiving at least $95 \%$ of the prescription dose), dose received by $2 \%$ of tar- get volume (D2), D95, D98, and homogeneity index (HI) proposed in ICRU-83 [9].

\section{Dosimetric characterization}

For this purpose, we designed and printed flat boluses with different thicknesses. The attenuation and scatter properties of the 3D printed (malleable "rubber-like" photopolymer, Agilus ${ }^{\odot}$ ) and commercial (poliorganosiloxano, eXaSkin ${ }^{\odot}$ ) boluses were physically evaluated for a 6-MV clinical photon beam on a water-equivalent RW3 slab phantom in three configurations: without a bolus, with a commercial bolus (5 and $10 \mathrm{~mm}$ thickness), and with six $3 \mathrm{D}$ printed boluses (2 to $10 \mathrm{~mm}$ thickness), thus obtaining nine measures. We then selected the 5- and 10-mm 3D boluses for comparison with the commercial bolus. The irradiations were performed delivering 300 monitor units (MU), with a dose rate of $400 \mathrm{MU} / \mathrm{min}$, using a $10 \times 10 \mathrm{~cm}^{2}$ open field with 0 -degree gantry angle at $1000 \mathrm{~mm}$ skin-to-source distance (SSD). A Gafchromic EBT3 film (International Specialty Products, Wayne, NJ) were cut $(2 \times 3 \mathrm{~cm})$ and placed below the boluses providing depth dose measurements. Measurement films were scanned 24 hours after irradiation using a desktop flat-bed transmission Epson Expression Scanner 10000 XL (Epson, Long Beach, CA). The film scanner was operated with a resolution of 72 dpi in the 48-bit red-green-blue (RGB) mode.

\section{Physical measurements}

To evaluate the calculation algorithm AAA for the 3D bolus, we irradiated a field of $10 \mathrm{~cm} \times 10 \mathrm{~cm}$ with $300 \mathrm{MU}$ and the gantry at $90^{\circ}$ to get the per-

Table 1. Dosimetric parameters of 6 plans (prescribed dose: $50 \mathrm{~Gy}$ ): 2 without bolus, 1 with commercial bolus, and 3 with three-dimensional (3D) printed bolus

\begin{tabular}{|c|c|c|c|c|c|c|}
\hline Parameter & No bolus & No bolus* & $\begin{array}{l}\text { Commercial } \\
\text { bolus }\end{array}$ & $\begin{array}{l}\text { 3D bolus } \\
(0.5 \mathrm{~cm})\end{array}$ & $\begin{array}{l}\text { 3D bolus } \\
(1 \mathrm{~cm})\end{array}$ & $\begin{array}{l}\text { 3D bolus } \\
(1 \mathrm{~cm})+\mathrm{gel}\end{array}$ \\
\hline $\mathrm{D}_{\max }[\mathrm{Gy}(\%)]$ & $60.5(121)$ & $59.4(118.8)$ & $54.2(108.4)$ & $55(110)$ & $51.7(103.4)$ & $51.7(103.4)$ \\
\hline $\mathrm{D}_{\text {mean }}[\mathrm{Gy}(\%)]$ & $45.3(90.6)$ & $52.3(104.6)$ & $50(100)$ & $50(100)$ & $50(100)$ & $50(100)$ \\
\hline $\mathrm{D}_{\min }[\mathrm{Gy}(\%)]$ & $7.8(15.6)$ & $40.2(80.4)$ & $45.2(90.4)$ & $44.1(88.2)$ & $47.8(95.6)$ & $47.9(95.8)$ \\
\hline $\mathrm{V}_{95 \%}(\%)$ & 55.5 & 95.3 & 100 & 99.6 & 100 & 100 \\
\hline $\mathrm{D}_{2 \%}[\mathrm{~Gy}(\%)]$ & $56.4(111)$ & 57 (114) & $52.4(104.8)$ & $52.5(105)$ & $51.1(102.4)$ & $51.1(102.4)$ \\
\hline $\mathrm{D}_{95 \%}[\mathrm{~Gy}(\%)]$ & $25.2(112.8)$ & 47.5 (95) & $49.2(98.4)$ & $48.7(97.4)$ & $49.3(98.6)$ & $49.3(98.6)$ \\
\hline $\mathrm{D}_{98 \%}[\mathrm{~Gy}(\%)]$ & $20.7(41.4)$ & $46.2(92.4)$ & $48.8(97.6)$ & $48.4(96.8)$ & $49.1(98.2)$ & $49.1(98.2)$ \\
\hline $\begin{array}{l}\text { Homogeneity } \\
\text { index** }\end{array}$ & 0.787 & 0.208 & 0.073 & 0.083 & 0.041 & 0.040 \\
\hline
\end{tabular}

*Virtual target volume without bolus excluding the $3 \mathrm{~mm}$ closest to the skin surface; ** Homogeneity index (HI) proposed in the International Commission on Radiation Units and Measurements Report $83\left[\left(\mathrm{D}_{2 \%}-\mathrm{D}_{98 \%}\right) / \mathrm{D}_{50 \%}\right]$. An HI of zero indicates that the absorbed-dose distribution is almost homogeneous 
cent depth dose (PDD) profile under the three following conditions in the Rando phantom: without a bolus, with a commercial bolus, and with the 3D boluses. Gafchromic EBT3 films (ISP Corporation, Wayne, NJ, USA) were cut and inserted along the horizontal direction in the phantom. After irradiation, the films were scanned in the RGB mode with an Epson 10000XL flatbed scanner according to the manufacturer recommendations for the film. The scanned images were analyzed with the use of the Omnipro I'mRT Version 1.7 (IBA Dosimetry, Schwarzenbruck, Germany) package. The calibration curves of the EBT3 film were determined in the red channel. A more detailed description of the film dosimetry with the use of the Gafchromic EBT films was provided in a previous study [10]. For the analysis, film dose measurements were smoothed applying a Gaussian filter $3 \times 3$ and the percentage of points that meet the gamma value $(5 \%, 3$ $\mathrm{mm})<1$ was obtained. The PDD profiles corresponding to the measured dose in the EBT film and the calculated dose in treatment planning system were compared.

\section{Results}

We fabricated the customized 3D bolus (Fig. 1). CT simulation indicated it fit the ear acceptably (Fig. 2). Due to the irregular shape of the outer ear anatomy, there was some air gap between the bolus and the phantom surface with the commercial bolus (Fig. 2). We observed that the target coverage was slightly better with the $3 \mathrm{D}$ bolus of $10 \mathrm{~mm}$ compared with the commercial one $\left(\mathrm{D}_{98 \%} 98.2 \% v s\right.$. 97.6\%; Tab. 1). The maximum dose was reduced by $6.6 \%$ with the $3 \mathrm{D}$ bolus and the minimum dose increased by $5.2 \%$ when comparing with the commercial bolus. In addition, the homogeneity index was better for the 3D bolus (0.041 vs. 0.073; Tab. 1).

Table 2 shows the measured doses with the $2-10$ mm thickness 3D-bolus as well as those with the 5-10 $\mathrm{mm}$ thickness commercial bolus. There was

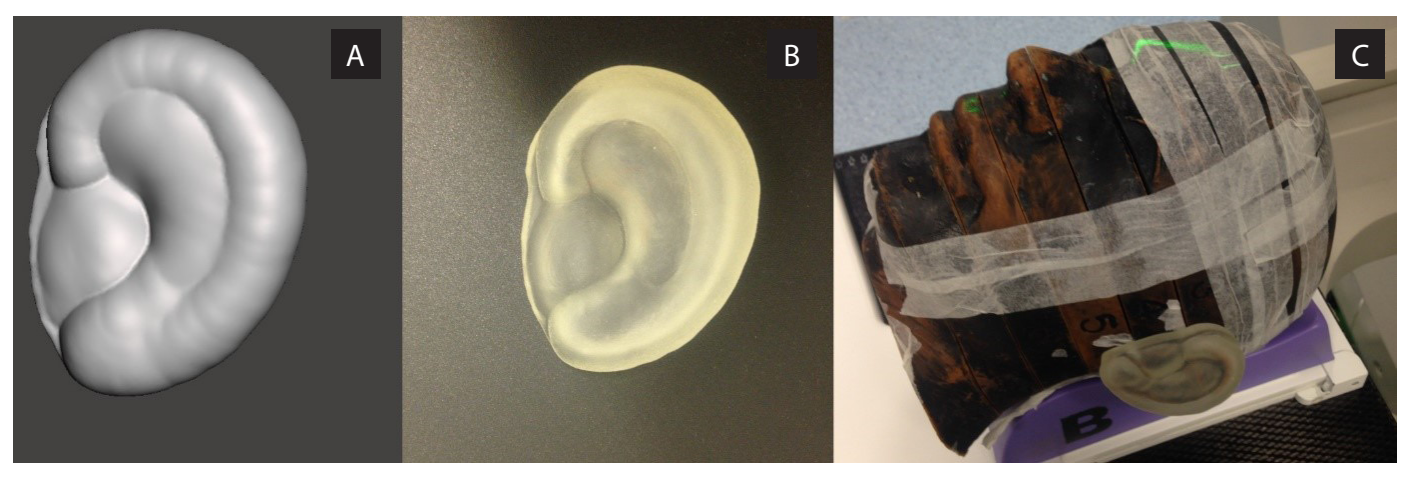

Figure 1. Three-dimensional (3D) printed customized bolus of the outer ear. A. View of the designed 3D bolus; B. Printed result obtained with malleable material; $\mathbf{C}$. Smulation scan setup of the bolus on the Rando phantom

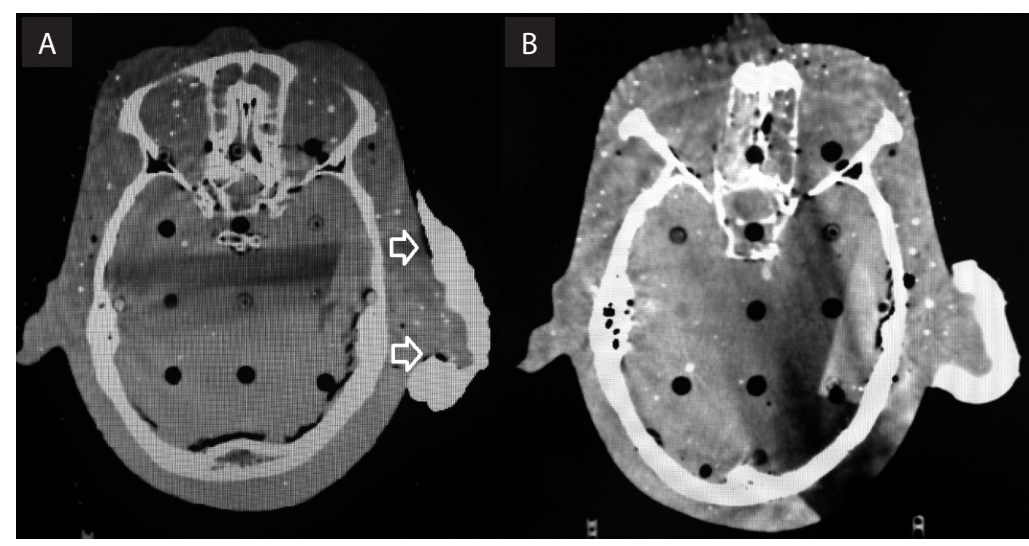

Figure 2. Computed tomography scan showing (A) commercial bolus, and (B) three-dimensional printed customized bolus for the irregular shape of the outer ear. Arrows indicate air gaps 
Table 2. Calculated doses with 2-10 mm thickness 3D-bolus and the measured doses with 5-10 mm thickness commercial bolus.

\begin{tabular}{|c|c|c|c|}
\hline Bolus type & Thickness [mm] & Mean dose [cGy] & Standard deviation [cGy] \\
\hline \multirow{7}{*}{ Three-dimensional printed } & 0 & 69.1 & 2.2 \\
\hline & 2 & 212.3 & 3.0 \\
\hline & 4 & 271.7 & 2.7 \\
\hline & 5 & 305.3 & 6.5 \\
\hline & 6 & 303.6 & 4.2 \\
\hline & 8 & 310.6 & 4.5 \\
\hline & 10 & 320.9 & 4.1 \\
\hline \multirow{2}{*}{ Commercial } & 5 & 318.1 & 6.3 \\
\hline & 10 & 313.0 & 6.4 \\
\hline
\end{tabular}

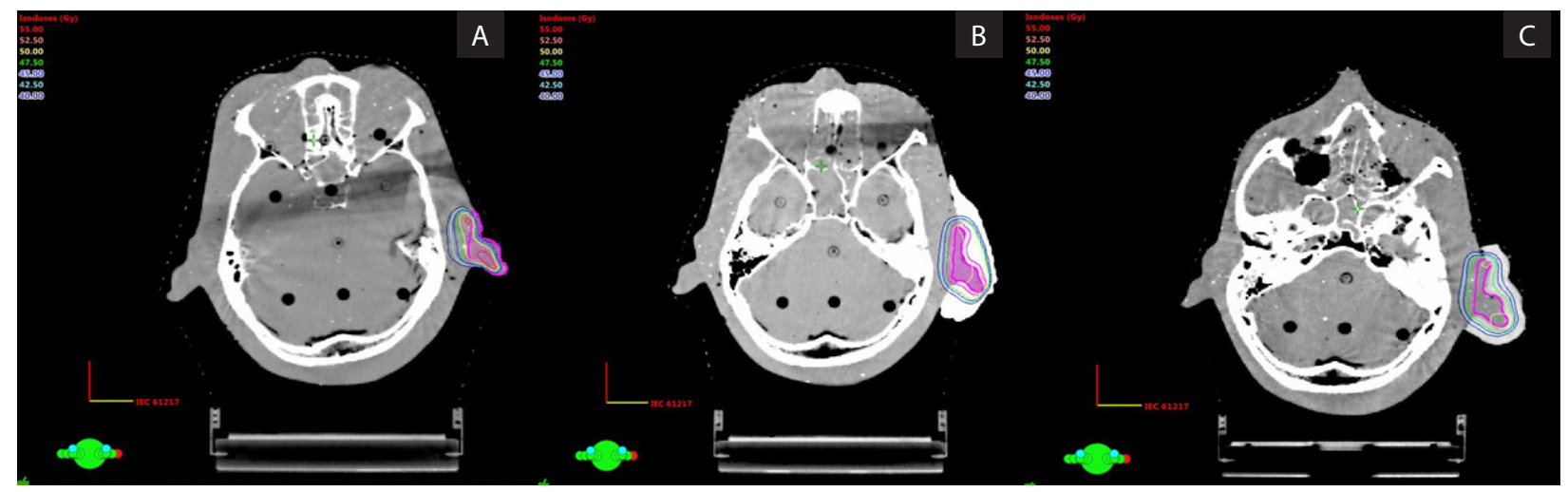

Figure 3. Isodose lines corresponding to the plans without $(\mathbf{A})$ and with the commercial $(\mathbf{B})$ and the three-dimensional printed customized $(\mathbf{C})$ bolus
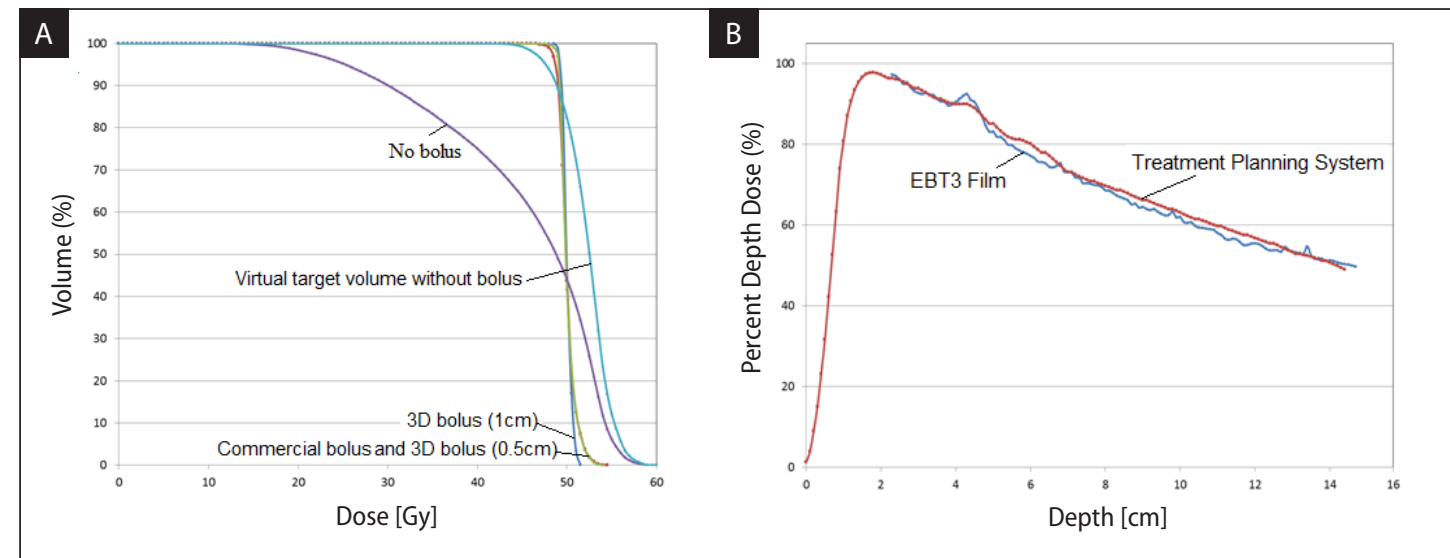

Figure 4. Dose volume histogram of the target corresponding to the plans with and without the bolus $(\mathbf{A})$ and the percent depth dose analysis with the use of Gafchromic EBT3 film (B)

a $4 \%$ and $2 \%$ difference between the $3 \mathrm{D}$-bolus and the commercial bolus when evaluating the 5 and $10 \mathrm{~mm}$ thickness, respectively.

Figure 3 shows the isodose lines corresponding to the plans with and without the bolus. We observe that the target coverage is better with the $3 \mathrm{D}$ bolus and it is similar compared with the commercial bolus. Figure 4A shows the dose-volume histogram of the ear with and without the 3D bolus, and Table 1 summarizes the relevant dosimetric parameters 
for all plans. Further, the PDD curves corresponding to the measured dose in the EBT film and the calculated dose in treatment planning system were comparable (Fig. 4B).

\section{Discussion}

Megavoltage photon RT penetrates through the skin to irradiate deep-seated tumors, with skin-sparing property. The build-up effect is considered a major benefit because of the reduction in skin toxicity. Hence, to treat superficial lesions, a commercial bolus is commonly used in such situations to increase the surface dose in radiation oncology units [11]. In case of an irregular surface region of a patient, unwanted air gaps under the bolus might occur between the bolus and patient skin due to the malleability of bolus material. It has been reported that an air gap of $4 \mathrm{~mm}$ causes a reduction of dose to the basal layer of approximately $0-4 \%$ depending on field size, angle of incidence and other patient specific parameters and a reduction of up to $10 \%$ could be seen at the basal cell layer for a $10 \mathrm{~mm}$ air gap [12]. Our pertinent findings can be summarized as follows. First, the target coverage was similar and even slightly better with the $3 \mathrm{D}$ bolus compared with the commercial one. Second, the maximum dose was reduced with the $3 \mathrm{D}$ bolus and the minimum dose increased when comparing with the commercial bolus. Finally, the homogeneity index was better for the 3D bolus.

Fujimoto et al. [13] customized a patient-specific $3 \mathrm{D}$ bolus using a $3 \mathrm{D}$ printing technique and evaluated its clinical feasibility for photon RT. The virtual target volume was delineated below the surface of the phantom in the vicinity of the nose. In the physical evaluation, the $3 \mathrm{D}$-bolus provided effective dose coverage in the build-up region, which was equivalent to the commercial-bolus. With regard to the clinical feasibility and in agreement with us, the air gaps were lesser with the 3D-bolus when compared to the commercial-bolus and the prescription dose could be delivered appropriately to the target volume. The 3D-bolus had potential use for air-gap reduction compared to the commercial-bolus and facilitated target-volume dose coverage and homogeneity improvement. In addition, there was not any dosimetric improvement when adding gel between the 3D-bolus and the ear surface. It seems that the air gaps between the body surface and the
3D bolus were not large enough to cause a lack of electronic balance. Hence, it is not necessary to use this gel to fill the virtual space after the $3 \mathrm{D}$ bolus application.

In algorithms that do not take heterogeneities into account, the calculation accuracy for commercial boluses tends to be lower. For instance, the Eclipse planning system uses a dose volume optimizer optimization algorithm for IMRT that does not optimize adequately in areas of low or high density. That could be the reason why dosimetric parameters with the $3 \mathrm{D}$ bolus are slightly better than those of the commercial high density bolus. The 3D bolus had a radiological density similar to water. Both the optimization algorithms and the calculation algorithms are precise in this setting. The planning system appropriately assigns the Hounsfield number to its density and the calculation algorithm is valid.

The design of a 3D bolus with the use of CT scans leads to unwanted radiation exposure of the patient [14]. To be able to make the bolus with little patient involvement and in minimal time, it has been proposed that the patient does not need to have a second CT done with the printed bolus for treatment planning [7]. The printed bolus density is overridden in Eclipse with a Hounsfield unit number of 260 . Then, the treatment plan can be calculated on the new CT using the same delivery parameters. Others [5] have proposed the use of a 3D surface scanner for the 3D bolus fabrication to reduce unnecessary radiation exposure. Park et al. [5] successfully fabricated a customized 3D bolus for a nose using a 3D surface scanner instead of a CT scanner. In comparison with the process based on the CT scan, the fabrication process based on a 3D surface scanner is simple and less time-consuming because this approach can skip several steps in the normal process from the contouring of the body surface to the generation of the STL file. The target presented in this report is challenging due to the very irregular surface of the ear. Although a $3 \mathrm{D}$ surface scanner could be used, the complex anatomy of the ear hinders the acquisition of images. The CT scanner improves overall image quality in this case. In addition, clinical information is crucial for the $3 \mathrm{D}$ bolus design in order to have a more customized therapy.

Our dosimetric analysis in this study showed that satisfactory target coverage could be achieved 
with the proposed 3D bolus. Moreover, our PDD analysis with EBT film confirmed that the bolus fabricated could increase the surface dose effectively, and this dose was comparable with that of a commercial bolus. This is in agreement with prior studies focused mainly on the nose $[5,15]$. Another benefit could be when the bolus should be replaced during the treatment for some reason [16]. The 3D bolus can be replaced fast and accurately reproducing the previous one, thus assuring equivalent treatment reproducibility.

\section{Conclusion}

We successfully fabricated a 3D bolus to cover a very irregular surface. The fabrication process was simple and fast. The bolus, made of malleable material, suitably fitted the surface, and the surface dose was sufficiently enhanced. The designed bolus is potentially useful for high-accuracy dose delivery through the reduction of unexpected air gaps in the case of irregularly shaped patient-skin surfaces. In addition, the 3D bolus can be replaced easily if necessary with the exact shape of the previous one. Thus, we believe that the use of a $3 \mathrm{D}$ bolus and malleable materials can be seriously considered for the fabrication of customized boluses for very irregular surfaces.

\section{Conflict of interest and Funding}

Authors declare that we do not have any financial support or relationships that may suppose conflict of interest.

\section{Ethical standards}

Research not involving human participants and/or animals. Informed consent is not required for this study.

\section{References}

1. Jung $\mathbf{N H}$, Shin $\mathrm{Y}$, Jung $\mathrm{IH}$, et al. Feasibility of normal tissue dose reduction in radiotherapy using low strength magnetic field. Radiat Oncol J. 2015; 33(3): 226-232, doi: 10.3857/roj.2015.33.3.226, indexed in Pubmed: 26484306.

2. Hogstrom K. Treatment Planning in Electron Beam Therapy. Front Radiat Ther Oncol.: 30-52, doi: 10.1159/000429576, indexed in Pubmed: 908420.

3. Guadagnolo BA, Zagars GK, Araujo D, et al. Outcomes after definitive treatment for cutaneous angiosarcoma of the face and scalp. Head Neck. 2011; 33(5): 661-667, doi: 10.1002/hed.21513, indexed in Pubmed: 20960566.

4. Tieu MT, Graham P, Browne L, et al. The effect of adjuvant postmastectomy radiotherapy bolus technique on local recurrence. Int J Radiat Oncol Biol Phys. 2011; 81(3): e165-e171, doi: 10.1016/j.jijrobp.2011.01.002, indexed in Pubmed: 21362579.

5. Park JW, Oh SeAn, Yea JiW, et al. Fabrication of malleable three-dimensional-printed customized bolus using three-dimensional scanner. PLoS One. 2017; 12(5): e0177562, doi: 10.1371/journal.pone.0177562, indexed in Pubmed: 28494012.

6. Valverde I, Gomez-Ciriza G, Hussain T, et al. Three-dimensional printed models for surgical planning of complex congenital heart defects: an international multicentre study. Eur J Cardiothorac Surg. 2017; 52(6): 1139-1148, doi: 10.1093/ejcts/ezx208, indexed in Pubmed: 28977423.

7. Burleson S, Baker J, Hsia AnT, et al. Use of $3 D$ printers to create a patient-specific 3D bolus for external beam therapy. J Appl Clin Med Phys. 2015; 16(3): 5247, doi: 10.1120/jacmp. v16i3.5247, indexed in Pubmed: 26103485.

8. Park K, Park S, Jeon MJ, et al. Clinical application of 3D-printed-step-bolus in post-total-mastectomy electron conformal therapy. Oncotarget. 2017; 8(15): 25660-25668, doi: 10.18632/oncotarget.12829, indexed in Pubmed: 27784001.

9. Bethesda MICoRUaM: ICRU Report 83.. Prescribing, Recording, and Reporting Photon-Beam IntensityModulated Radiation Therapy (IMRT). J ICRU. 2010; 10(1), doi: $10.1093 /$ jicru/ndq002.

10. Carrasco MA, Perucha M, Luis FJ, et al. A comparison between radiochromic EBT2 film model and its predecessor EBT film model. Phys Med. 2013; 29(4): 412-422, doi: 10.1016/j.ejmp.2012.05.008, indexed in Pubmed: 22738767.

11. Vyas V, Palmer L, Mudge R, et al. On bolus for megavoltage photon and electron radiation therapy. Med Dosim. 2013;38(3): 268-273, doi: 10.1016/j.meddos.2013.02.007, indexed in Pubmed: 23582702.

12. Butson $M$, Cheung T, Yu P, et al. Effects on skin dose from unwanted air gaps under bolus in photon beam radiotherapy. Radiat Measure. 2000; 32(3): 201-204, doi: 10.1016/ s1350-4487(99)00276-0.

13. Fujimoto K, Shiinoki T, Yuasa Y, et al. Efficacy of patientspecific bolus created using three-dimensional printing technique in photon radiotherapy. Phys Med. 2017; 38: 1-9, doi: 10.1016/j.ejmp.2017.04.023, indexed in Pubmed: 28610688.

14. Park JW, Yea JW, Park JW, et al. Three-dimensional customized bolus for intensity-modulated radiotherapy in a patient with Kimura's disease involving the auricle. Cancer Radiother. 2016; 20(3): 205-209, doi: 10.1016/j. canrad.2015.11.003, indexed in Pubmed: 27020714.

15. Kim SW, Shin HJ, Kay CS, et al. A customized bolus produced using a 3-dimensional printer for radiotherapy. PLoS One. 2014; 9(10): e110746, doi: 10.1371/journal. pone.0110746, indexed in Pubmed: 25337700.

16. Robar JL, Moran K, Allan J, et al. Intrapatient study comparing 3D printed bolus versus standard vinyl gel sheet bolus for postmastectomy chest wall radiation therapy. Pract Radiat Oncol. 2018; 8(4): 221-229, doi: 10.1016/j. prro.2017.12.008, indexed in Pubmed: 29452866. 